\title{
Aportes y eXperiencias para la Educación en Derechos Humanos "CAMinOS PARA LA INTEGRACIÓN SOCIAL"
}

\section{Contributions and experiences to the Human Rights Education "Paths for the social integration"}

\author{
Mariana Blengio Valdés*
}

\begin{abstract}
RESUMO
A Educação em Direitos Humanos abrange diferentes desafios que podem ser abordados no campo universitário através da inclusão dos diversos coletivos. Neste marco se integram aos processos de capacitação em Direitos Humanos participantes que provém de instâncias do Ministério do Interior do Uruguai e da Escola Nacional de Polícia. O Ciclo de Educação ofertado pela Universidad de la República propicia atividade de extensão educativa no processo de formação dos cadetes e como forma de atualização e sensibilização para aqueles que desempenham funções no referido Ministério. Iniciativas como a Campanha "Um Livro e um Disco para os Cárceres" e o Programa de "Visitas aos Memoriais" constituem experiências de destaque.
\end{abstract}

Palavras-chave: educação; direitos humanos; polícia.

\begin{abstract}
The Education in Human Rights faces different challenges that are possible to be discussed since the university field, through the inclusion of diverse collectives. In this path, processes of qualification in Human Rights offered to different instances of the Uruguay Interior Ministry and the National School of Policy are integrated. The Annual Cycle of Education of the Unesco Chair in Human Rights University of the Republic propitiates educative extension activity in the process of official's formation and it is an updating and responsive form for those who have functions in the referred Ministry. Initiatives as the Campaign "a Book and a Record for the Jails" and the Program of "Visits to Memorials" constitute prominent experiences.
\end{abstract}

Keywords: education; human rights; police.

\section{RESUMEN}

La Educación en Derechos Humanos plantea diferentes desafíos que se abordan desde el campo universitario a través de la inclusión de los diversos colectivos. En este marco se integran a los procesos de capacitación en Derechos Humanos participantes que provienen de las dependencias del Ministerio del Interior de Uruguay y de la Escuela Nacional de Policía. El Ciclo de Educación impartido en la Universidad de la República oficia de extensión educativa para el proceso de formación de los cadetes y como forma de actualización y sensibilización para quienes se encuentran en funciones dentro del referido Ministerio. Iniciativas como la Campaña "Un Libro y un Disco para la Cárcel" y el Programa de "Visitas a los Memoriales" constituyen experiencias de destaque.

Palabras-clave: educación; derechos humanos; policía.

\footnotetext{
Doctora en Derecho y Ciencias Sociales. Profesora Adscripta de Derechos Humanos de la Universidad de la República. Coordinadora de la Cátedra UNESCO de Derechos Humanos de la Universidad de la República. ius@netgate.com.uy

www.rau.edu.uy/universidad/ddhh
} 


\section{Universidad y Derechos Humanos}

Las Universidades constituyen espacios privilegiados para fortalecer los desarrollos y cambios en materia cultural. Como centros de estudio e investigación, las instituciones de nivel terciario pueden albergar en su seno las diferentes corrientes de pensamiento que circundan en nuestras sociedades en un diálogo plural basado en la tolerancia y la libre expresión de ideas.

Es así como desde su interior, los movimientos universitarios generan transformaciones de diversa índole, cuyos efectos permiten transferir con el paso del tiempo sus experiencias a la comunidad, contribuyendo a fortalecer las transformaciones.

Bajo esta premisa, la Cátedra UNESCO de Derechos Humanos de la Universidad de la República viene desarrollando variados programas de integración cívica entre diversos actores sociales. Las actividades tienen tres ejes. Estos son: capacitación, investigación y promoción de los Derechos Humanos. En el área de capacitación se siguen adelante los Ciclos Anuales de Educación en Derechos Humanos. En el año 2007 se llevó a cabo la VIII Edición del mismo. Participaron un promedio de 500 asistentes. Así también se desarrollan Talleres puntuales sobre temas de actualidad. En el campo de investigación, la Cátedra UNESCO ha realizado diversos aportes, muchos de los cuales se han publicado. Entre ellos se destacan: Dignidad Humana, publicación que incluye el aporte académico de prestigiosos especialistas de Uruguay. El libro Uruguay y la Declaración Universal incorpora los antecedentes que determinaron la participación de Uruguay en el proceso de adopción del documento universal de 1948. A la fecha se han editado dos números de la Revista Derechos Humanos. En ella se integran aportes de actualidad de especialistas nacionales y extranjeros.
En el campo didáctico se ha innovado con investigaciones que derivaron en la edición de CD Roms interactivos. Entre ellos puede reseñarse: Declaración Universal de Derechos Humanos; Las voces de la Declaración; Aportes interdisciplinarios para la Educación en Derechos Humanos: Genocidio y Tortura. En el campo de la promoción la Cátedra ha diseñado la convocatoria de cuatro Concursos de Ensayos. Los mismos versaron sobre: Derecho Humano a la Paz; Declaración Universal de Derechos Humanos; Genocidios del Siglo XX y Tortura. La participación de estudiantes universitarios en dichos Concursos ha sido relevante. En los dos primeros resultaron ganadores jóvenes de la Universidad Nacional Autónoma de México y la Universidad de Buenos Aires. En los Concursos de Genocidios y Tortura fueron premiados estudiantes de la Universidad de la República. En el convocado en el año 2006 sobre Genocidios en el Siglo XX, recibió Mención de Honor un trabajo presentado por estudiantes de la Escuela Militar de Uruguay que refería al Genocidio en Camboya.

\section{Ciclo Anual de Educación en Derechos Humanos}

El trabajo se inició en el año 2000 y su desarrollo ha sido paulatino. Desde un inicio la Cátedra UNESCO de Derechos Humanos ha trabajado en capacitación en el área de extensión universitaria. En virtud de ello, llevan a cabo durante el año diversas actividades centralizadas en un Ciclo de Educación que tiene por meta el abordaje de temáticas relativas a los Derechos Humanos desde diferentes enfoques. Con esta actividad se pretende dar una visión con perspectivas centradas en el respeto a los Derechos de la persona humana y el reconocimiento intrínseco de su Dignidad. El Ciclo Anual de Educación en Derechos 
Humanos ha buscado entonces forjar una visión integradora del abordaje educativo para introducir las diferentes temáticas y disciplinas, con una lectura académica en clave de Derechos Humanos.

Múltiples desafíos se agolpan a la hora de diseñar una propuesta universitaria que pueda albergar todas las corrientes intelectuales, así como también los diferentes enfoques que emanan de las áreas de estudio. En este marco, la consigna radica en integrar las disciplinas universitarias bajo el común denominador de la comprensión del respeto a los Derechos de la persona y el aprendizaje interactivo de las obligaciones que subyacen en toda Democracia y que habilitan la creación y fortalecimiento del Estado de Derecho.

Así fueron nucleándose a través de las propuestas del calendario ideado en forma anual, diversos colectivos que se vieron motivados por las iniciativas plasmadas. Su integración es testimonio de una necesidad básica de comprensión de la universalidad del ser humano en el desarrollo formativo de la persona.

\section{Participación de funcionarios policiales y cadetes de la Escuela Nacional de Policía}

A partir de esta propuesta abierta se fue integrando un número importante de participantes. Entre ellos, estudiantes de las diferentes áreas universitarias, docentes de todos los niveles de enseñanza, magistrados, fiscales, miembros o integrantes de organizaciones no gubernamentales, funcionarios estatales. ${ }^{1}$ Desde el año 2004 a la fecha, participan en el Ciclo un número destacado de personas que provienen de diferentes dependencias del Ministerio del Interior, así como también cadetes de la Escuela Nacional de Policía.

La presencia de los cadetes en las actividades de la Cátedra UNESCO significó un mutuo aprendizaje. En primer lugar, una aproximación de la Dirección de la propia Escuela a través de la promoción de las actividades del Ciclo Anual a las diferentes generaciones de cadetes. Esta motivación significó un importante paso en la apertura educativa de la Institución, lo que contribuyó en la búsqueda perseguida por la Cátedra UNESCO de la eliminación de los estereotipos sociales que derivan en la exclusión de determinados colectivos, fruto de trágicas y dramáticas circunstancias históricas.

Sin embargo, la presencia de los cadetes, en forma gradual en los diferentes Ciclos, ha demostrado el interés y la avidez de éstos, por el conocimiento como forma de complementación de los estudios en el ámbito policial. Desde una perspectiva diferente que les permite aproximarse a autores, expositores y docentes provenientes de muy variadas áreas del conocimiento.

El Ciclo Anual al cual los cadetes y otros funcionarios policiales asisten, oficia de complemento a los estudios que poseen o que se llevan a cabo en la Escuela Nacional de Policía uruguaya. Un abordaje que promueve el conocimiento de temáticas que provenientes de la Historia, Literatura, Sociología, Medicina, Arquitectura, entre otras disciplinas, busca reunir a través del denominador común de la enseñanza, la comprensión de los fenómenos sociales.

1 Es de destacar que en virtud a la modalidad semi presencial ideada para el Ciclo asisten al mismo personas que provienen del interior del país, así como también del exterior, especialmente Argentina y Brasil. En el año 2003 se recibieron tres pasantes del Instituto de Derechos Humanos de la Universidad de Lyon en Francia. En 2004 se integró como pasante un funcionario de la Defensoría de Derechos Humanos de Colombia. 


\section{Declaración de interés ministerial}

Esta presencia permanente en la cual el conjunto de asistentes se confunden en un todo que presencia, pregunta, aprende y critica desde el conocimiento y la sana comprensión los aportes que emanan de los especialistas, determinó que el Ministerio del Interior declarara de interés ministerial las actividades del Ciclo Anual en el año 2007.² Así también la propia Escuela Nacional de Policía, en el mismo año, resuelve conceder el auspicio al VIII Ciclo de Educación en Derechos Humanos por considerar que:

La participación en el mencionado Ciclo, favorece la capacitación de los alumnos de esta casa de estudios, dado que opera como complemento a la asignatura que se imparte en el primer curso, además de permitir al resto del cuerpo de cadetes y oficiales de este centro docente, participar activamente en una política que la Dirección de esta Escuela viene impulsando desde años atrás, consustanciados de la importancia del tratamiento del tema de los Derechos Humanos en la formación de los futuros Oficiales de la Policía Nacional, para brindar el mejor y mas eficaz servicio a la población de nuestro país. ${ }^{3}$

A modo de ejemplo podemos reseñar que las temáticas desarrolladas en los diferentes Ciclos fueron muy diversas. La dinámica consiste en el dictado de una exposición mensual. A lo largo del año se verifican 8 sesiones. El Ciclo se proyecta de mayo a diciembre, finalizando generalmente el día 10 de ese mes en conmemoración de un nuevo Aniversario de la Declaración Universal de Derechos Humanos de Naciones Unidas de 1948. ${ }^{4}$

Fueron abordados en las sesiones, entre otros temas: La búsqueda de la verdad ante el terrorismo de Estado; El arte, fundamento de la cultura de paz; El Comisionado Parlamentario ante los desafíos de la realidad carcelaria; El ejercicio del derecho a la participación ciudadana; El arte, los monumentos y la identidad nacional; Derechos Humanos y Misiones de Paz; Los desafíos de la Bioética en el siglo XXI; La protección judicial de los Derechos Humanos a cien años de la creación de la Suprema Corte de Justicia; Historias para el Nunca Más. Persecución durante la dictadura militar; El Defensor del Vecino de Montevideo; Trata de Blancas y Prostitución. Ayer y Hoy. ${ }^{5}$

\section{Campañas y programas educativos}

Las actividades del Ciclo son el eje central de la tarea anual. A ello se han agregado otras propuestas complementarias. Entre ellas desarrollaremos las siguientes: Campaña Un Libro y un Disco para la Cárcel y Programa educativo Visita a los Memoriales.

La Campaña Un Libro y un Disco para la Cárcel nace del impulso fraterno basado en la necesidad de retribuir la apertura con la cual los asistentes del Ciclo fueron recibidos por las autoridades de las diferentes Instituciones Carcelarias del país en innumerables visitas realizadas a los centros. En el marco del trabajo anual

\footnotetext{
Resolución del Ministerio del Interior de 17 de abril de 2007.

Resolución de la Escuela Nacional de Policía de 8 de mayo de 2007.

4 Los Ciclos Anuales de Educación en Derechos Humanos fueron declarados de interés educativo por el Ministerio de Educación y Cultura en los años 2005, 2006 y 2007 por Resoluciones de fecha 19 de julio 2005, 27 de junio 2006 y 17 de mayo 2007.

5 Temas incluidos en las sesiones de los Ciclos Anuales del Año 2006 y 2007. Puede verse también la reseña de los Ciclos de años anteriores en la Memoria de la Cátedra UNESCO de Derechos Humanos de la Universidad de la República, Vivir en clave de Derechos Humanos 1999 - 2004, editada por la Cátedra UNESCO de Derechos Humanos de la UDELAR en diciembre de 2004.
} 
y en coordinación con la Dirección Nacional de Cárceles del Ministerio del Interior, se habilitan a grupos de asistentes al Ciclo a realizar visitas educativas a las Instituciones Carcelarias. En ellas los asistentes que como se ha visto provienen de muy diversas áreas, pueden acercarse a la problemática carcelaria desde una experiencia real que les acerca e interioriza con la temática.

Las visitas son acompañadas en muchos casos de charlas que las propias autoridades facilitan dentro del propio Penal, así como también la posibilidad de dialogar con los reclusos.

La instrumentación de la campaña ha permitido que año a año la Cátedra UNESCO reúna un número importante de libros que son donados a las Bibliotecas de las Instituciones Carcelarias en señal de colaboración y agradecimiento. La propuesta fue ampliada en el año 2005 con la inclusión de discos donados a las Bibliotecas de las diferentes Cárceles, con el fin de poder colaborar con las actividades lúdicas de reclusos y funcionarios de los Penales. ${ }^{6}$

El Programa de Visita a los Memoriales fue una propuesta diferente. Comienza el trabajo a través del abordaje académico en los diferentes Ciclos de temáticas que vinculaban la Arquitectura y los Derechos Humanos tal cual se ha visto. Así pudo realizarse una aproximación del arte de la construcción desde la perspectiva de los Derechos Humanos, y a través de esto, el conocimiento de los diferentes procesos que determinaron trágicas coyunturas históricas, cuya envergadura es difícil resumir. Entre ellos: el Genocidio del Pueblo Armenio; el Holocausto y la Muerte, Desaparición y Tortura de miles de personas durante la Dictadura Militar que azotó el Uruguay durante los años 1973 a 1984.
La iniciativa comprende la visita didáctica a diferentes Memoriales existentes en la ciudad de Montevideo: Plaza Armenia, Holocausto y Detenidos Desaparecidos. En las recorridas se trata de hacer "visible lo invisible", buscando a través del relato histórico dar vuelo al sentido artístico que se plasmó en los monumentos. ${ }^{7}$ Para lograr que la Memoria de los hechos ocurridos se trasmita como una esperanza de paz en la arquitectura diseñada en forma simbólica por quienes crearon allí el testimonio de miles de historias para el Nunca Más.

\section{Conclusión}

El trabajo realizado por la Cátedra UNESCO de Derechos Humanos de la Universidad de la República, en este sentido, alberga a la fecha resultados positivos que permiten avizorar un tímido cambio en la concepción de un Uruguay ideado desde la reconciliación y la búsqueda de la Paz, basada en la Justicia como valores esenciales de la Democracia. Una reconciliación que nace desde la apertura cívica. Que no evite ni excluya temáticas ni colectivos, sino que albergue en el conjunto universal de los estudios académicos la perspectiva plural de la Humanidad.

Sin embargo, el real desafío de esta experiencia radica en que la misma perdure. No ya como un relato de las actividades impartidas o realizadas, sino como una incorporación al obrar de cada uno de quienes las compartimos.

\footnotetext{
6 En el año 2004 y 2005 la donación de libros y discos se destinó a la Cárcel de Mujeres de Cabildo. En el año 2006 la donación se hizo a la Cárcel Departamental de Colonia, "Piedra del Indio". En el año 2007 se hicieron dos donaciones, una a la Cárcel Departamental de Rocha y otra al Penal de Libertad. El total de ejemplares donados por la Cátedra UNESCO asciende a más de 1000.

7 Puede verse al respecto el registro fotográfico y la fundamentación teórica de la propuesta en el CD ROM "Aportes Interdisciplinarios para la Educación en Derechos Humanos", editado por la Cátedra UNESCO de Derechos Humanos de la Universidad de la República en diciembre de 2007.
} 
Constituye hoy, meta y fin, la posibi- a la Dignidad de todo ser humano. Será allí, en la lidad de que esta experiencia madure en el coyuntura histórica, que el futuro arroje cuando ser interior de quienes las han realizado, que se podrá verificar realmente si el augurio del hoy permitan incorporar la fraternidad en el trato se constituye en el presente del mañana. humanitario y la perdurable vocación de respeto

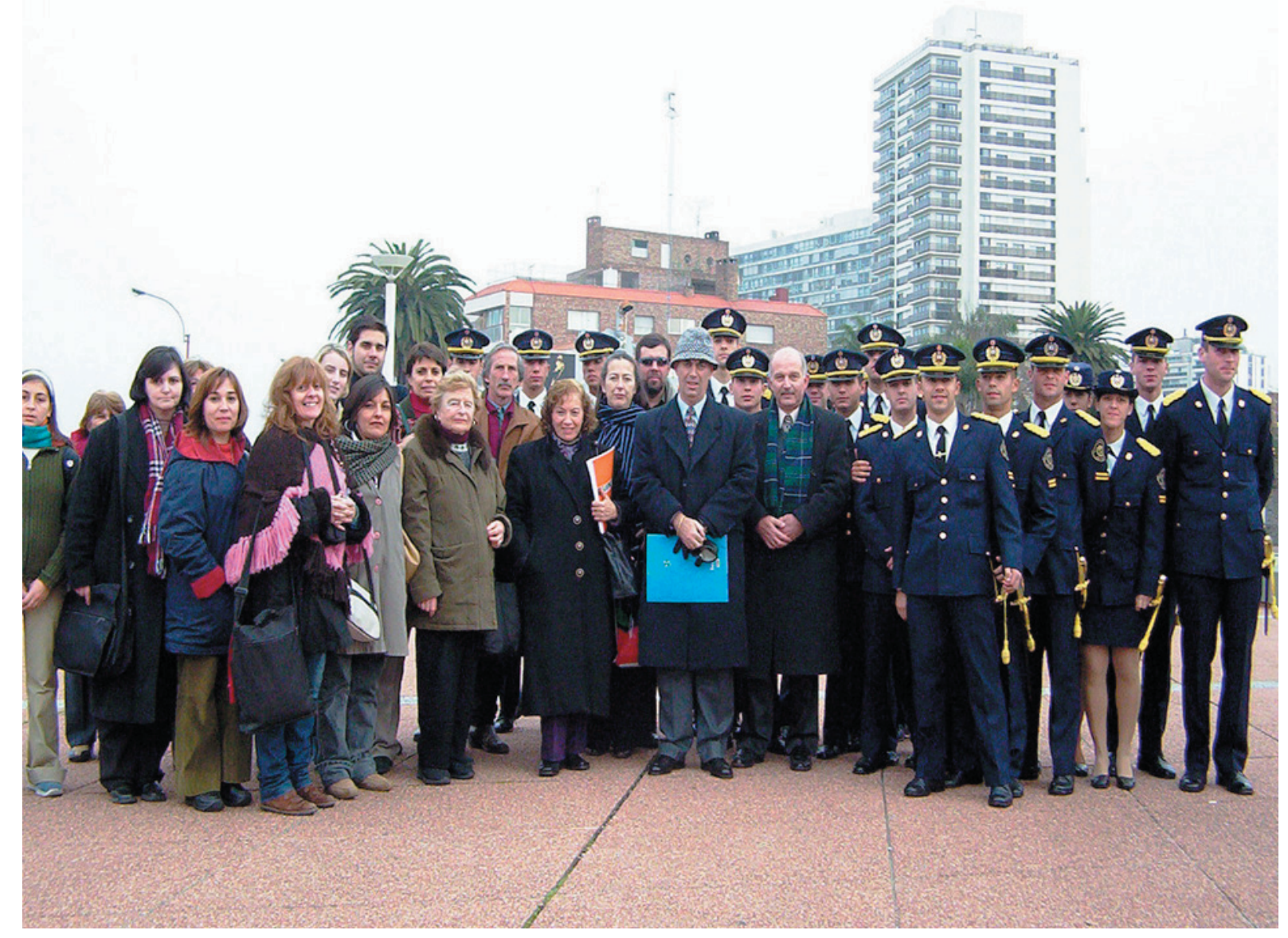

En la plaza se alberga el diseño de una gigante Aguila de frente al mar.

El monolito que se encuentra en dicho Memorial incluye la siguiente leyenda: Uruguay, recibe de tus hijos adoptivos este símbolo de gratitud y lealtad.

FIGURA 1 - VISITA AL MEMORIAL DE LA PLAZA ARMENIA 


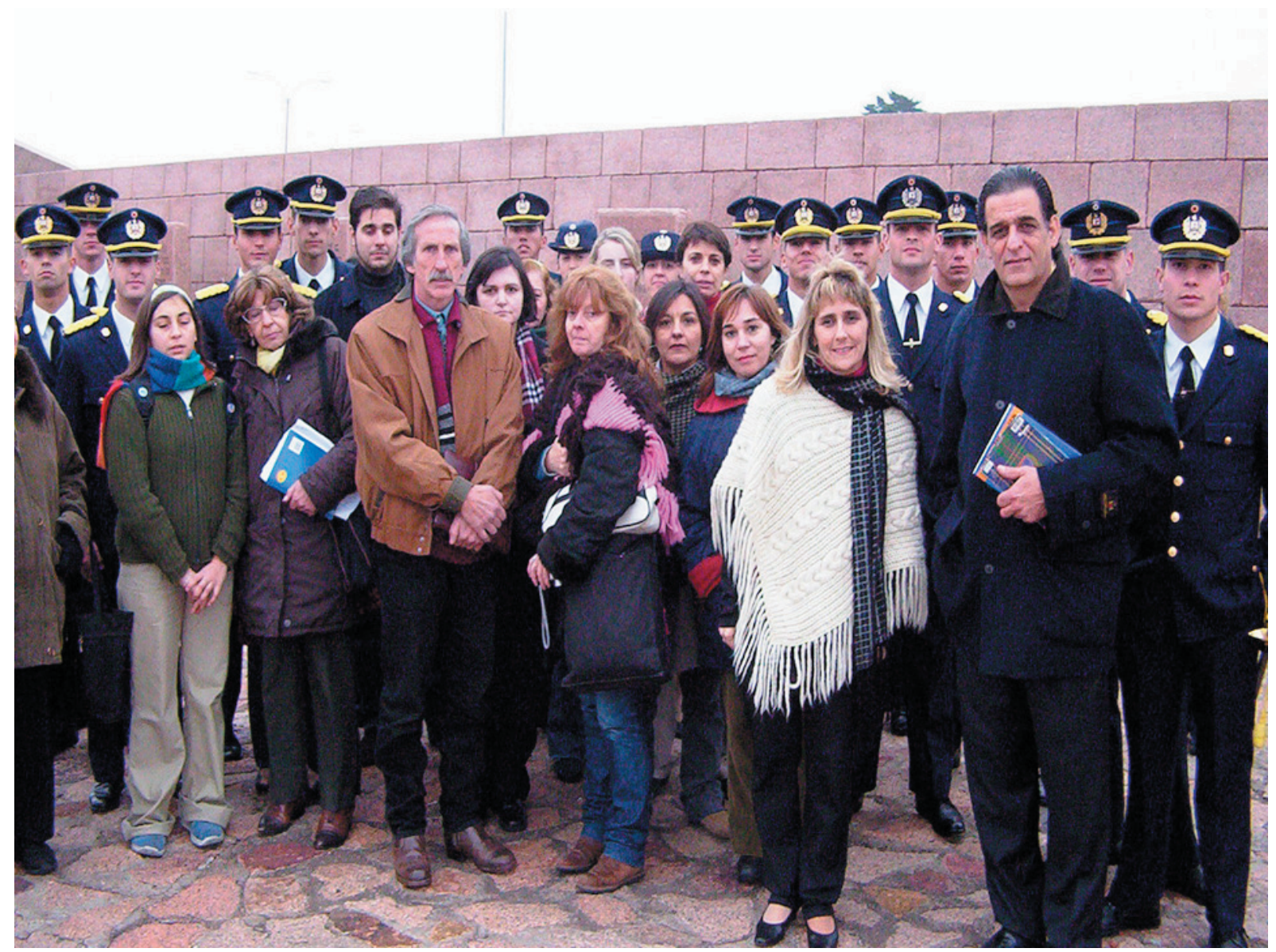

Ubicado frente al río (Río de la Plata), tiene un doble simbolismo: los trozos de granito se imponen al agua, simbolizan la apertura, así como también la llegada de los inmigrantes. Su recorrida está marcada por las vías del tren que conducen a la muerte en los Campos de Exterminio y Concentración Nazis. Así también la presencia del muro cuya ruptura permite visualizar cuatro lápidas colocadas en fila con las siguientes inscripciones. Siete veces cae el justo y siete veces volverá a levantarse (Proverbios 24:18); Elegid siempre la vida y el bien pues la elección está en vuestras manos (Maimónides); En el recordar está la redención (Baal Shem Tov) y A la tristeza queremos que la acompañe la esperanza (Elie Wiesel).

FIGURA 2 - VISITA AL MEMORIAL DEL HOLOCAUSTO DEL PUEBLO JUDÍO 


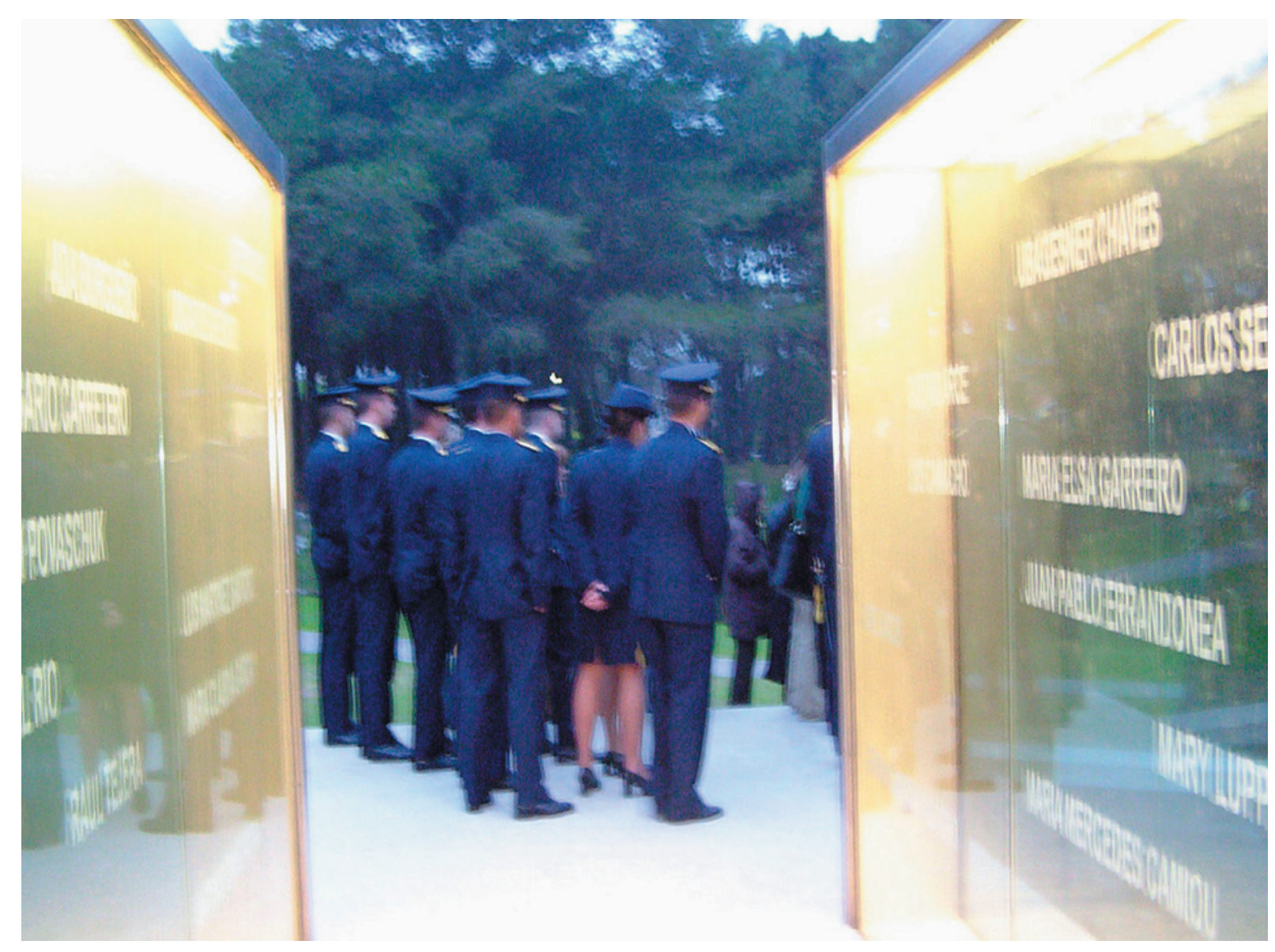

Según expresa la Comisión Pro Memorial de los Detenidos Desaparecidos: El Memorial implica un paso más en la búsqueda de mecanismos de reparación de las víctimas de la dictadura. Se trata de coadyuvar en el proceso de construcción de la memoria histórica dando testimonio de un período de nuestra historia. En este proceso de reflexión colectiva se inserta la idea de este memorial que procura desde todos los sectores de la sociedad, el camino de la paz a través del esclarecimiento de los hechos. El Memorial trasciende la coyuntura, dejando un testimonio de reparación en particular de la verdad individual y concreta sobre los Detenidos Desaparecidos.

FIGURA 3 - VISITA AL MEMORIAL DE DETENIDOS DESAPARECIDOS EN EL CERRO DE MONTEVIDEO

Texto recebido em 14 ago. 2007 Texto aprovado em 19 fev. 2008 